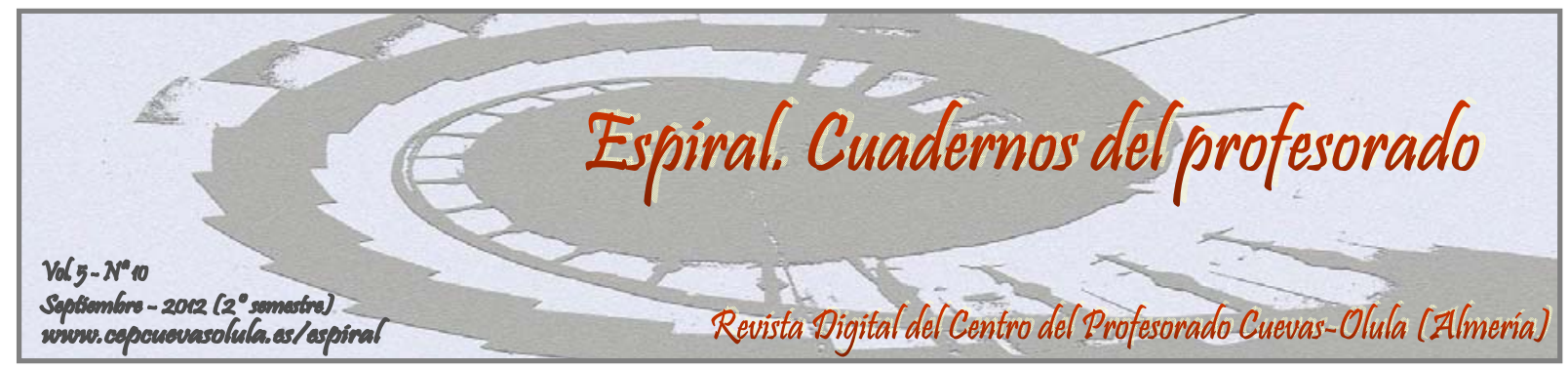

\title{
COMPETENCIAS PROFESIONALES EN EDUCACIÓN FÍSICA Y NECESIDADES EDUCATIVAS
}

\section{THE PROFESSIONAL COMPETENCES IN PHYSICAL EDUCATION AND EDUCATIONAL NEEDS}

\author{
Antonio Baena-Extremera ${ }^{(1)}$ y Antonio Granero-Gallegos ${ }^{(2)}$
}

(1) Facultad de Ciencias del Deporte, Universidad de Murcia, Murcia, España

(2) Centro del Profesorado Cuevas-Olula, Junta de Andalucía, España

RESUMEN: El proceso de Bolonia y la necesidad de homologar los títulos en el Espacio Europeo de Educación Superior, ha supuesto la creación de las carreras universitarias desde el enfoque competencial, que hasta ahora, estaban en un segundo plano. El objetivo de este artículo ha sido realizar un pequeño análisis de lo que ha supuesto y debe suponer, la creación de los grados de Educación Física, desde dicho enfoque hacia la creación de un estilo de vida activo en los alumnos. Pensamos que crear un enfoque curricular competencial de los estudios puede ser el modo de asumir la responsabilidad social de educar para una vida saludable, tras las investigaciones que cada día nos muestran las necesidades de la población actual. Para ello, hacen falta ciertos cambios tanto en los planes de estudios, como en la formación de los futuros docentes.

Palabras clave: Educación Física, grados, estilo de vida activo.

ABSTRACT: With The Bologna process, and the need to standardize the qualifications in the European Higher Education, has created the university from focusing competence, so far, were in the background. The aim of this paper is to make a short analysis which has led and must involve the creation of physical education degrees from the approach to the creation of an active lifestyle in students. We think we create curricular approach competence of the studies can be how to take social responsibility to educate for a healthy life, following investigations show that every day needs of the current population. Therefore, certain changes are needed in both the curriculum and in the teaching of future teachers.

Key words: Physical Education, degrees, active lifestyle.

Baena-Extremera, A. y Granero-Gallegos, A. (2012). Competencias profesionales en Educación Física y necesidades educativas. Espiral. Cuadernos del Profesorado, 5(10), 105-109. Disponible en: http://www.cepcuevasolula.es/espiral.

Fecha de recepción: 25/02/2012

Fecha de aceptación: 14/06/2012
Enviar correspondencia a: abaenaextrem@um.es 


\section{1.- INTRODUCCIÓN}

La investigación en torno a las competencias profesionales no es nada nuevo, pues ya al inicio de los años 70 se comenzó a hablar de las mismas, dentro de los enfoques tecnológicos y conductistas del entrenamiento del profesorado, según tendencias de modelos tales como el CBTE (Competency Based Teacher Education) (Navio, 2005, p. 216). Posteriormente, se especula sobre la competencia profesional, pero desde el punto de vista del mundo laboral, del mundo empresarial, etc. Actualmente, la evolución de la concepción de competencia es mucho más complejo de lo que se puede creer, debido a su polisemia. No sólo se entienden como conjunto de saberes, capacidades o habilidades que se aplican en la resolución de problemas vinculados a un perfil profesional determinado, sino que además tiene connotaciones psicológicas y sociales (Romero, 2009). Tomando como ejemplo a Tejada (2005, p.7), se entiende por Competencia Profesional "un conjunto de conocimientos, procedimientos y actitudes combinados, coordinados e integrados, en el sentido que el individuo ha de saber hacer $y$ saber estar para el ejercicio profesional".

Desde el Espacio Europeo de Educación Superior (EEES) y en la necesidad de homologar todos los títulos ante los nuevos retos planteados desde la sociedad del conocimiento y la globalización, se urge al sistema educativo universitario para que responda con más eficacia a las necesidades del mercado de trabajo y, por supuesto, en relación a la realidad social cada vez más cambiante. Esto supone que, los planes de estudios de las titulaciones que se elaboren nuevos deberán buscar desde ahora una formación basada en la adquisición de competencias, teniendo como referente que éstas serán las que orienten el perfil profesional del título (González y Wagenaar, 2003). Por otro lado, el sobrepeso de la población estudiantil española (Moreno et al., 2005) y su bajo nivel de condición física (Ortega et al., 2005), hacen de esto una necesidad social a la que los futuros planes de estudios de Educación Física (EF), deben atender.

En el sistema educativo existe una amplia experiencia en diseñar currículos a partir de competencias. Por ejemplo, se ha utilizado de manera destacada en Formación Profesional y en la Formación de Adultos, planificando el aprendizaje de manera que se aproxime su formación al mundo del trabajo lo máximo posible. Ahora las competencias se han llevado a la mayoría de los niveles educativos, tanto obligatorios como no, debido, entre otros aspectos, a la urgencia por conseguir resultados y una elevación de los niveles académicos. A esto hay que sumarle que poco a poco hemos ido apreciando como la evaluación y el control de la educación por parte de los gobiernos (TIMSS, PIRLS, ALL o PISA) en el contexto de la educación globalizada coherente con las necesidades del mercado, han hecho que sean otro de los factores influyentes en el despliegue actual de las competencias profesionales. Todo lo anterior, ha conllevado que se termine inclinando la balanza en la utilización de las competencias dentro del sistema educativo (Bolivar, 2008).

En el caso de la universidad, la incorporación de las competencias se debe a varios motivos. Por un lado, es entendido como un modo de acercar la universidad a la sociedad y al ámbito laboral, y como tal constituye una recomendación recogida en el Informe Universidad 2000 de la CRUE para la profesionalización de la formación universitaria. Por otro, es entendido como un modo de reclamar una enseñanza más práctica y útil para los estudiantes. Refuerza el planteamiento de una formación que se diseña mirando al aprendizaje y al alumnado en varios sentidos: trabajar con aprendizaje significativos, unir la teoría con la praxis, buscar una formación integral de los alumnos, etc.

Además de los motivos expuestos, a partir de Bolonia (1999) urgió la necesidad de hacer patente las competencias en la formación universitaria, principalmente ligada al concepto de economía del conocimiento, la innovación tecnológica y las demandas del mercado (Escudero, 2008). Llegaron a estos niveles educativos a través de los Libros Blancos de Titulaciones realizados por las diversas comisiones creadas por ANECA (Agencia Nacional de Evaluación de la Calidad y Acreditación) con el propósito de establecer inicialmente las directrices de la reforma de las titulaciones (Escudero, 2008). Así, incorporar el concepto de competencia en los niveles universitarios implica intensificar el carácter integral y la responsabilidad social de la formación universitaria con la creación de los perfiles profesionales. 


\section{2.- LAS COMPETENCIAS PROFESIONALES EN EDUCACIÓN FÍSICA}

En el diseño de las titulaciones según las directrices marcadas por los libros blancos mencionados, los créditos que los alumnos cursan deben servir para atender a todas y cada una de las salidas profesionales de dicha carrera. Obviamente, luego queda por ver la especialización que los alumnos puedan cursar dentro del grado y en forma de postgrado. Un ejemplo claro lo podemos ver en el caso del Grado de Maestro con mención a EF. En esta carrera, los créditos ECTS destinados a la formación específica de EF deberían garantizar la capacitación y cualificación profesional del maestro en Educación Primaria para impartir la EF (Romero, 2009). Teniendo en cuenta lo especificado, las nuevas demandas sociales que tiene la escuela afectan de manera directa y decisiva al trabajo del docente, su función ya no es únicamente transmisora de contenidos y si más centrada en el aprendizaje y en la persona como ser humano interacciona con el entorno social y cultural en el que vive (Romero, 2009). Y además, como bien afirma Romero (2007), posibilitar un buen desempeño en la enseñanza de esta área curricular, en cuanto a la importancia social que pueda tener en la conformación de hábitos y estilos de vida y en la transmisión de una cultura corporal tan importante para la sociedad en la que vivimos.

Así, si tenemos en cuenta que de los 240 créditos ECTS que conforma el Título de Grado de Maestro, la estructura se conforma con 60 créditos para el módulo de formación básica, 100 créditos para módulo didáctico disciplinar y 50 créditos para el módulo del practicum (incluyendo el trabajo fin de Grado), quedando solamente 30 créditos para poder conformar la mención en EF (Romero 2009). ¡Solamente 30 créditos!

Siguiendo la Orden ECI/3857/2007, por la que se establecen los requisitos para la verificación de los títulos universitarios oficiales que habiliten para el ejercicio de la profesión de Maestro en Educación Primaria, dentro del apartado 5 titulado "Planificación de las enseñanzas", recoge que en estas enseñanzas podrán proponerse menciones cualificadoras, entre 30 y 60 créditos europeos, adecuadas a los objetivos, ciclos y áreas de la Educación Primaria, según lo establecido en los artículos 17, 18, 19 y 93 de la LOE. ¿Solamente 30 créditos, son suficientes?

Pero es más, teniendo en cuenta en el Grado de maestro con mención a EF y según lo establecido en las enseñanzas mínimas para la Educación Primaria (según el Real Decreto 1513/2006), se deberán abordar conocimientos y habilidades relacionados con:

- El desarrollo de las capacidades perceptivo-motoras.

- El desarrollo de las habilidades motrices.

- La expresividad a través del cuerpo y el movimiento: Actividades físicas artísticoexpresivas

- La actividad física saludable, proporcionando actitudes y hábitos de actividad física para lo largo de la vida y de unos estilos de vida, como fuente de bienestar.

- Relativos al juego motor y a las actividades deportivas como manifestaciones culturales de la motricidad humana.

Quizás es demasiado, para tan poco, o simplemente, es poco, tanto en contenidos como en créditos. Pues bien, habiendo lo que hay, desde nuestro punto de vista si pretendemos crear hábitos para una vida activa y saludable, debemos atender sobre todo a las competencias específicas. Por ejemplo:

- Promover hábitos de actividad física y estilos de vida saludables en los escolares.

- Crear una relación necesaria entre la actividad física con los hábitos de higiene y de salud.

De hecho, Romero (2009) concluye en su trabajo que el docente en EF debe tener la capacidad de promover hábitos y estilos de vida saludables, estableciendo conexión entre la actividad física y los cuidados corporales (hábitos de higiene y salud).

Destacar además, que en las investigaciones realizadas por Romero (2009), Romero, Zagalaz, Romero y Martínez (2011) con alumnos de magisterio, como la competencia de "Promover hábitos y estilos de vida saludables..." es una de las más valoradas. En segundo lugar destaca la Competencia 
de "Relación necesaria entre la actividad física con los hábitos de higiene, posturales y de salud, como contenido específico del área de EF y como contenido transversal”.

Igualmente, en el caso de la titulación en Ciencias de la Actividad Física y del Deporte, tras los estudios realizados por la ANECA para la elaboración de los Libros Blancos, se obtiene como las competencias más valoradas por los Docentes en EF y profesionales de la Enseñanza, la Capacidad de transmitir Actitudes y Valores....

Se aprecia como es un aspecto determinante de interés social la aportación que se pueda hacer desde la escuela y la EF como área curricular en la promoción de la Actividad Física y la Salud (Romero, 2007).

\section{3.- CONCLUSIONES}

Como conclusiones, nos gustaría terminar con algunas aportaciones y recopilaciones que podemos encontrar en la literatura al respecto y que creemos bien merece la pena:

- Teniendo en cuenta los resultados de los Proyectos Avena y/o Helena (entre otros), existe una clara tendencia social del alumnado en relación a los hábitos y práctica de actividad física.

- Plantear un enfoque curricular competencial puede ser el modo de asumir la responsabilidad social de educar para una vida saludable.

- Para ello, las competencias profesionales deben transferirse al alumno de nuestras universidades para que sea consciente y capaz de adoptar un estilo de vida activo. $\mathrm{Y}$ a su vez, para que este lo transmita a sus futuros alumnos.

- Investigaciones realizadas por Granero, Ruiz y García (2009), afirman que la población comprendida entre 15 y 29 años, opinan que las clases de EF recibidas en el centro educativo sirvieron para crear hábitos de vida saludables.

- La figura del docente juega un papel importante en la adquisición de un estilo de vida saludable.

- Este planteamiento supondrá renovar las metodologías docentes, adaptándolas a las nuevas necesidades sociales.

- La especificidad y la proximidad entre el profesional de la salud y el "cliente-alumno" hace que los estudiantes de EF tengan que disponer de otras competencias específicas diferentes a las que se dan en otras salidas profesionales, como: de relación y atención al cliente, habilidades sociales, iniciativa y liderazgo, gestión comercial, coordinación y dirección de grupos de trabajo.

\section{4.- REFERENCIAS BIBLIOGRÁFICAS}

Bolívar, A. (2008). El discurso de las competencias en España: educación básica y educación superior. Red U. Revista de Docencia Universitaria, "Formación centrada en competencias(II)”, [en línea]. Disponible en: http://www.redu.m.es/Red_U/m2. Consulta [2012,7 de Septiembre].

CRUE (2000). Declaración de Bolonia: adaptación del sistema universitario español a sus directrices, [en línea]. Disponible en: http://www.crue.org/. Consulta [2012, 7 de Septiembre].

Escudero, J.M. (2008, Junio). Las competencias profesionales y la formación universitaria: posibilidades y riesgos. Red U. Revista de Docencia Universitaria, "Formación centrada en competencias(II)" [en línea]. Disponible en: http://www.redu.m.es/Red_U/m2. Consulta [2012, 1 de Septiembre].

Granero, A., Ruiz, F.J., y García, M.E. (2009). Opinión de la educación física recibida. En F.J. Ruiz, M.E. García, y M. Pierón (coord.) Actividad física y estilos de vida saludable: análisis de los determinantes de la práctica en adultos (pp.151-162). Sevilla: Wanceullen.

González, J., y Wagenaar, R. (2003). Tuning Educational Structures in Europe. Final Report-Phase One. [en línea]. Disponible en: http://www.relint.deusto.es/TUNINGProject/index.htm. Consulta [2012, 7 de Junio]. 
Moreno, L.A., Mesana, M.I., Fleta, J., Ruiz, J.R., González-Gross, M.M., Sarría, A., Marcos, A., Bueno, M., y Grupo AVENA. (2005). Overweight, obesity and body fat composition in Spanish adolescents. The AVENA Study. Annanls of Nutrition Metabolic, 29, 49(2), 71-76.

Navio, A. (2005). Propuestas conceptuales en torno a la competencia profesional. Revista de Educación, 337, 213-234.

Orden ECI/3857/2007, de 27 de diciembre, por la que se establecen los requisitos para la verificación de los títulos universitarios oficiales que habiliten para el ejercicio de la profesión de Maestro en Educación Primaria. BOE, $\mathrm{n}^{\circ} 312$ de 29 diciembre de 2007.

Ortega F.B., Ruiz J.R., Castillo M.J., Moreno L.A., González-Gross M., Wärnberg J., Gutiérrez A., y Grupo AVENA. (2005). Bajo nivel de forma física en los adolescentes españoles. Importancia para la salud cardiovascular futura. (Estudio AVENA). Revista Española de Cardiología, 58(8), 898-909.

Real Decreto 1513/2006, de 7 de diciembre, por el que se establecen las enseñanzas mínimas de la Educación primaria. BOE, $\mathrm{n}^{\circ} 293$ de 8 de Diciembre de 2006.

Romero, C. (2007). Delimitación del campo didáctico de la Educación Física y de su actividad científica. Revista Currículum y Formación del Profesorado, [en línea] 11(2). Disponible en: http://www.ugr.es/local/recfpro/rev112ART1.pdf. Consulta [2012, 2 de Junio].

Romero, C. (2009). Definición de módulos y competencias del maestro con mención en Educación Física. Revista Internacional de Medicina y Ciencias de la Actividad Física y el Deporte, [en línea] 9(34), 179200. Disponible en: http://cdeporte.rediris.es/revista/revista34/artcompetencias124.htm. Consulta [2012,1 de Julio].

Romero C., Zagalaz, M.L., Romero, M., y Martínez-López, E.J. (2011). Importancia de las competencias profesionales de los Maestros en Educación Física expresadas por los estudiantes. Retos. Nuevas tendencias en Educación Física, Deporte y Recreación, 19, 62-68.

Tejada, J. (2005). El trabajo por competencias en el prácticum: cómo organizarlo y cómo evaluarlo. Revista Electrónica de Investigación Educativa, [en línea], 7 (2). Disponible en: http://redie.uabc.mx/vo7no2/contenido-tejada.html. Consulta [2012,14 de Julio]. 